\title{
Emerging Solutions in Big Data and Cloud Technologies for Mobile Networks
}

\author{
Vijayakumar Varadarajan ${ }^{1} \cdot$ Venkataraman Neelanarayanan $^{1} \cdot$ Ron Doyle $^{2} \cdot$ Imad Fakhri Al-Shaikhli ${ }^{3} \cdot$ Sven Groppe $^{4}$
}

Published online: 14 February 2019

(C) Springer Science+Business Media, LLC, part of Springer Nature 2019

With the emergence of the recent internet and mobile technologies, the massive user-generated data has started driving our daily lives. The popularity and wider utilization of Cloud Technologies and Big data have attracted global researchers in solving the complex and heterogeneous real-world problems. The deployment of cloud technologies for big data problems is much helpful in addressing the scalability and security issues. The fusion of the big data and cloud technologies has created new opportunities for the development of effective solutions to the key research domains such as the Internet of Things, health care, travel, smart city, energy, and agriculture. Data analytics, trust management, massive data management are few of the well-established research areas which have gained increased importance over the past years. The technological advancements enrich the creativity of researchers which helps in attaining efficient adaptive solutions. Such rich adaptive solutions open the way to new dimensions of security violations in mobile networks.

This Special Issue aims to bring together researchers to publish state-of-art research findings in Big Data and Cloud Technologies for Mobile Networks, focusing on both theoretical

Vijayakumar Varadarajan
vijayakumar.v@ vit.ac.in
Venkataraman Neelanarayanan
neelanarayanan.v@ vit.ac.in
Ron Doyle
rdoyle@alumni.duke.edu
Imad Fakhri Al-Shaikhli
imadf@ iium.edu.my
Sven Groppe
groppe@ifis.uni-luebeck.de
Vellore Institute of Technology, Chennai, India
Continuous Delivery Architecture, CA Technologies, New York, NY,
USA
International Islamic University, Malaysia, Gombak, Malaysia
University of Lubeck, Lubeck, Germany

Vijayakumar Varadarajan

Venkataraman Neelanarayanan

neelanarayanan.v@vit.ac.in

Ron Doyle

Imad Fakhri Al-Shaikhli

imadf@iium.edu.my

Sven Groppe

Vellore Institute of Technology, Chennai, India

2 Continuous Delivery Architecture, CA Technologies, New York, NY,

3 International Islamic University, Malaysia, Gombak, Malaysia

4 University of Lubeck, Lubeck, Germany and applied techniques. We expect the papers of the special issue to serve as valuable references for a large audience from both academia and industry. After a stringent peer-review process, this special issue features eight selected papers with high quality.

The first article titled "Efficient User Profiling Based Intelligent Travel Recommender System for Individual and Group of Users" presents Activity and Behavior induced Personalized Recommender System (ABiPRS) as a hybrid approach to predict persuasive POI recommendations [1]. The proposed ABiPRS is designed to support travelling user by providing effective list of POIs as recommendations. Further, the authors have developed a novel hybridization approach for aggregating recommendations from multiple RSs to improve the effectiveness of recommendations and the proposed approaches are evaluated on the real-time large-scale datasets of Yelp and TripAdvisor. The second paper, "E-Health Cloud Security Using Timing Enabled Proxy Re-Encryption" analyzes the security issues in the E-Health cloud and presents a planning empowered intermediary re-encryption method to defeat the security issues [2]. The develop technique will allow only limited access rights to an authorized agent to access the records for a specific time period and it will use a searchable encryption and proxy Re-encryption techniques.

The third paper entitled "Secure Remote User Mutual Authentication Scheme with Key Agreement for Cloud Environment" demonstrates the security limitations of a password based authentication scheme, and show that the existing scheme is still vulnerable to forgery and offline password guessing attacks and it is also unable to provide user anonymity, forward secrecy and mutual authentication [3]. The authors present a new secure authentication scheme and proved that the proposed scheme is invulnerable to various attacks together with attacks observed in the analyzed scheme through both rigorous formal and informal security analysis. The next article "Energy-Aware Fault-Tolerant Dynamic Task Scheduling Scheme for Virtualized Cloud Data Centers" describes the developed dynamic task assignment and scheduling scheme as the energy-aware fault-tolerant dynamic scheduling scheme (EFDTS), to co-ordinately optimize resource utilization and energy consumption with a 
fault tolerant mechanism [4]. Authors have designed an elastic resource provisioning mechanism in the context of faulttolerance to improve resource utilization and energy efficiency. Furthermore, authors also developed a migration policy improve resource utilization and energy efficiency simultaneously.

The fifth article titled "Price Versus Performance of Big Data Analysis for Cloud Based Internet of Things Networks" makes detailed discussions on the issues of handling Big Data from an operational perspective in this new cloud based IoT network architecture [5]. The authors have proposed an optimization model to address the price versus performance while carrying out Big Data analysis in the cloud based IoT networks. The next paper entitled "A Performance Evaluation of Resilient Server with a Self-Repair Network Model" presents the performance evaluation of a resilient server that we built by combining virtualization technology and a self-repair network model in which represents the in-VMI [6]. The experiments conducted by the authors have revealed the performance of their proposed method in terms of low performance losses and high service availability.

The seventh paper entitled "Two-Server 3D ElGamal Diffie-Hellman Password Authenticated and Key Exchange Protocol Using Geometrical Properties" presents cutting edge tetrahedron (3D) based two-server Password Authenticated Key Exchange (PAKE) protocol using ElGamal and DiffieHellman (DH) mechanism [7]. The authors have analyzed and implemented the mechanism using geometrical shape based properties - circumcenter $(\omega)$ and the angle between the medians $(\theta)$. The last article "A Deep Learning Spatiotemporal Prediction Framework for Mobile Crowdsourced Services" presents a deep learning-based framework to predict crowdsourced service availability spatially and temporally [8]. The authors have introduced a novel two-stage prediction model based on historical spatio-temporal traces of mobile crowdsourced services and validated the effectiveness of the proposed framework through multiple experiments.

Acknowledgements The guest editors would like to thank all reviewers for their efforts in reviewing manuscripts submitted to this special issue. We also thank the Edit-in-Chief, Dr. Imrich Chlamtac for his supportive guidance during the entire process. We would like to thank the entire editorial team for their continuous support. We would also extend our special thanks to our Management and Administrative Teams of VIT India, IIUM Malaysia, CA Tech USA, UOL Germany, and our worldwide friends who had supported morally and motivated us to bring this successful Special Issue Live.

Publisher's Note Springer Nature remains neutral with regard to jurisdictional claims in published maps and institutional affiliations.

\section{References}

1. Logesh R, Subramaniyaswamy V, Vijayakumar V, Li X (2018) Efficient User Profiling Based Intelligent Travel Recommender System for Individual and Group of Users. Mobile Networks and Applications. https://doi.org/10.1007/s11036-018-1059-2
2. Vijayakumar V, Priyan MK, Ushadevi G, Varatharajan R, Manogaran G, Tarare PV (2018) E-Health Cloud Security Using Timing Enabled Proxy Re-Encryption. Mobile Networks and Applications. https:// doi.org/10.1007/s11036-018-1060-9

3. Karuppiah M, Das AK, Li X, Kumari S, Wu F, Chaudhry SA, Niranchana R (2018) Secure Remote User Mutual Authentication Scheme with Key Agreement for Cloud Environment. Mobile Networks and Applications. https://doi.org/10.1007/s11036-0181061-8

4. Marahatta A, Wang Y, Zhang F, Sangaiah AK, Tyagi SKS, Liu Z (2018) Energy-Aware Fault-Tolerant Dynamic Task Scheduling Scheme for Virtualized Cloud Data Centers. Mobile Networks and Applications. https://doi.org/10.1007/s11036-018-1062-7

5. Meerja KA, Naidu PV, Kalva SRK (2018) Price Versus Performance of Big Data Analysis for Cloud Based Internet of Things Networks. Mobile Networks and Applications. https://doi.org/10.1007/s11036018-1063-6

6. Winarno I, Ishida Y, Okamoto T (2018) A Performance Evaluation of Resilient Server with a Self-Repair Network Model. Mobile Networks and Applications. https://doi.org/10.1007/s11036-018$1103-2$

7. Kumari KA, Sadasivam GS (2018) Two-Server 3D ElGamal DiffieHellman Password Authenticated and Key Exchange Protocol Using Geometrical Properties. Mobile Networks and Applications. https:// doi.org/10.1007/s11036-018-1104-1

8. Said AB, Erradi A, Neiat AG, Bouguettaya A (2018) A Deep Learning Spatiotemporal Prediction Framework for Mobile Crowdsourced Services. Mobile Networks and Applications. https://doi.org/10.1007/s11036-018-1105-0

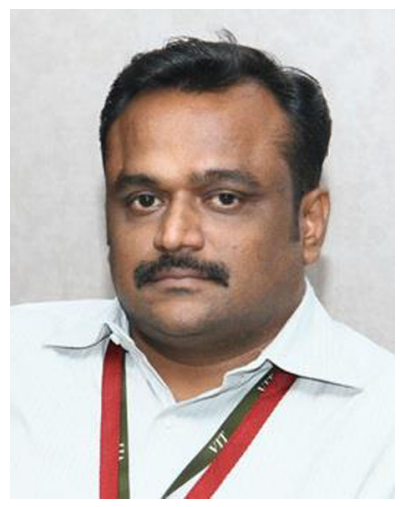

Vijayakumar Varadarajan is currently a Professor and an Associate Dean for School of Computing Science and Engineering at Vellore Institute of Technology, Chennai, India. He has more than 18 years of experience including industrial and institutional. He also served as a Team Lead in industries like Satyam, Mahindra Satyam and Tech Mahindra for several years. He has completed Diploma with First Class Honors. He has completed BE CSE and MBA HRD with First Class. He has also completed ME CSE with First Rank Award. He has completed his PhD from Anna University in 2012. He has published many articles in national and international level journals/conferences/books. He is a reviewer in IEEE Transactions, Inderscience and Springer Journals. He has initiated a number of international research collaborations with universities in Europe, Australia, Africa, Malaysia, Singapore and North America. He had also initiated joint research collaboration between Vellore Institute of Technology and various industries. He serves as an Editor-in-Chief for EAI Endorsed Transactions on Cloud Systems journal. He is also the Guest Editor for reputed journals of Inderscience, Springer and IGI Global. He also organized several international conferences and special sessions in USA, Vietnam, Africa, Malaysia and India including IEEE, ACSAT, ISRC, ISBCC, ICBCC etc. His research interests include computational areas covering grid computing, cloud computing, computer networks, cyber security and big data. He received his university - level Best Faculty Award for 2015-2016. He is also a member of several national and international professional bodies including EAI, BIS, ISTE, IAENG, CSTA, IEA etc. 


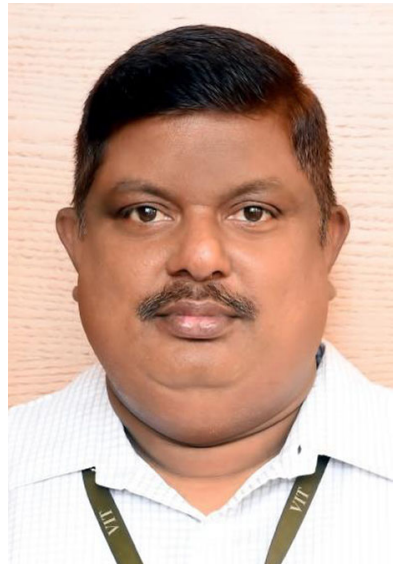

Venkataraman Neelanarayanan received his Master of Science in Computer Science from Madurai Kamaraj University, India in 1995 and $\mathrm{PhD}$ from IT University of Copenhagen, Denmark in 2012. Currently, he is an Associate Professor at VIT University, Chennai, India. Before joining VIT University he has worked as a Scientist at Centre for Advanced Computing (CDAC), India and as a Lecturer in Madurai Kamaraj University, India and its affiliated institutions. His areas of research include distributed computing such as grid and cloud computing, contextaware computing, network management and security, XML-based security technologies and e-communities.

He has initiated a number of international research collaborations with universities in Europe, Australia and South Korea as a Research Group Coordinator and Chief Investigator at VIT University. He was instrumental for initiating joint research collaboration between VIT University and industries such as CDAC and DLink. He has published more than 40 papers in various peer-reviewed international conferences and journals. He has organised various national workshops, international conference and symposium. Currently, he is serving as the overall coordinator for research groups at School of Computing Science and Engineering, VIT University, Chennai and six students are pursuing their $\mathrm{PhD}$ under his guidance. He is also the Guest Editor for few journals in Inderscience, Springer and IGI Global. He is editorin-chief for EAI Transactions on Cloud Systems. He is a member, Editorial Board, International Journal of Scientific Research in Computer Science and Engineering, and International Journal of Applied Science and Engineering, New Delhi Publishers. He received the research award in VIT University for the year 2015 for his achievements, exemplary commitment, dedication and motivation towards research publication during 2015-16. He is also a member of several national and international professional bodies including ISTE, IAENG, CSTA, SDIWC, IEA, ISTICC, EAI, WASET and IACSIT.

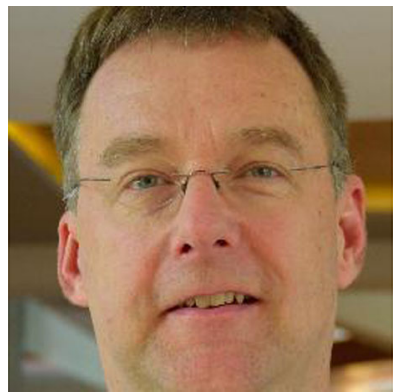

Ron Doyle is division-wide lead architect for enterprise and cloud application design/delivery, focusing on public SaaS, hybrid cloud and onpremise software products. The technical focus is all aspects of cloud and on-premise application/infrastructure architecture, services, APIs, containerization and DevOps. He is a member of the Board of Directors of the Eclipse Foundation. Before the Broadcom acquisition of $\mathrm{CA}$ Technologies, he was Vice President of Continuous Delivery Portfolio Architecture. He drove cross-product integration and product delivery models (SaaS and on-prem) for products in modeling, functional and performance testing, service virtualization and orchestration. Previously, he was a Distinguished Engineer for DevOps transformation across the IBM cloud development organization, focusing on both strategy and hands-on initiatives to address the organization, tools and methodologies needing to enable world-class service delivery. He has been a technical executive, project lead, designer, architect and developer on large scale projects, working with small and large customers at every level of the business. Previously he served as the technical lead for a cross-company organization focusing on key technical and strategic issues across IBM Cloud. His areas of interest include distributed systems and managed resource sharing, focusing on virtualization and cloud computing. He led the IBM involvement in the Open Virtualization Format (OVF) standard in the DMTF. He also spend time working on university accreditation for computer science programs. He has been a program evaluator and team chair for a number of years and currently on the ABET Computing Accreditation Commission Executive Committee and he is a Representative Director for the CSAB Board of Directors.

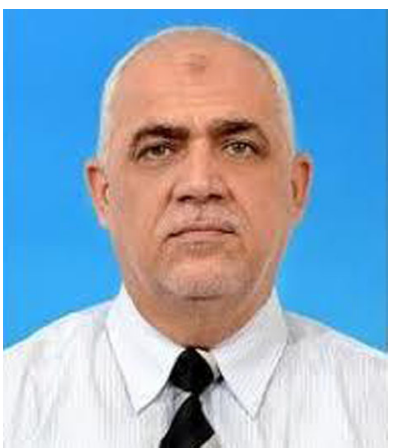

Imad Fakhri Al-Shaikhli is an IEEE senior member, obtained his BSc (Hon) in Mathematics, MSc in Computer Science from Iraq, and $\mathrm{PhD}$ degree from Pune University, India, 2000. In 2003 he was appointed as the head of department of computer information systems at Alrafidain University College until 2005 Then he joined Gulf universityBahrain January 2006 and appointed as the founding Dean of the college of computer engineering and sciences, during this period he introduced the CCNA certificate to be part of the curriculum which had a strong impact on the students career. In November 2010 he joined IIUM at the Dept. of Computer Science/ kulliyyah of Information and Communication Technology. He received the best teacher award in 2011. He is the editor in chief of JACSTR (international Journal on Advanced Computer Science and Technology Research) since 2011 till now and IJPCC international journal since 2015, and the general chair of the international conference on Advanced Computer Science Applications and Technologies) since 2012 till now. He obtained a US patent for his work with his PhD student on smart traffic light with accident detection system on 2nd Dec 2014. Prof. Imad has published more than 200 articles, conference papers, and book chapters in addition to three books. In addition, he secured more than 10 research grants. He supervised and graduated more 30 PG students. Presently Prof. Dr. Imad is a Professor at the Department of Computer Science and Head of Research at the Kulliyyah of Information and Communications Technology, the International Islamic University Malaysia (IIUM) since 1st November 2013.

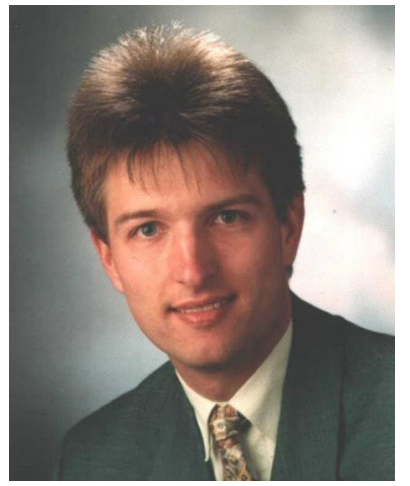

Sven Groppe earned his diploma degree in Informatik (Computer Science) in 2002 and his Doctor degree in 2005 from the University of Paderborn. He earned his habilitation degree in 2011 from the University of Lübeck. He worked in the European projects B2BECOM, MEMPHIS, ASG and TripCom. He was a member of the DAWG W3C Working Group, which developed SPARQL. He was the project leader of the DFG project LUPOSDATE, an opensource Semantic Web database, and one of the project leaders of two research projects, which research on FPGA acceleration of relational and Semantic Web databases. He is also the chair of the Semantic Big Data workshop series, which is affiliated with the ACM SIGMOD conference (so far 2016 to 2018), and of the Very Large Internet of Things workshop in conjunction with the VLDB conference in 2017 and 2018. His research interests include databases, Semantic Web, query and rule processing and optimization, Cloud Computing, peer-to-peer (P2P) networks, Internet of Things, data visualization and visual query languages. 